und Medien, verdeutlicht insbesondere anhand von Fragen der Regulierung des Internet und des elektronischen Handels. Zu den Verdiensten der Studie kann gezählt werden, dass das Themenfeld nicht nur umfassend und detailliert beschrieben, sondern auch thematisch gut strukturiert wurde. Neben den Skizzen und Analysen der existierenden Regulierungskonzepte sind vor allem die zahlreichen Vorschläge und Denkanstöße, wie sie in Zukunft gestaltet sein sollten, von Nutzen. Seinen Übersichtscharakter erhält das Buch dadurch, dass analytische Diskussionen, wie z.B. über die genannten ökonomischen Charakteristika des Mediamatik-Bereichs (Netzwerkeffekte, steigende Skalenerträge etc.), sich nicht im Detail verlieren. Sie werden vielmehr nur in soweit geführt, wie es für die nachfolgenden Erörterungen der Konzepte einer Mediamatik-Politik von Nöten ist. Dementsprechend sind die Analysen allerdings auch nicht erschöpfend, und so stellt sich zum Beispiel die Frage, ob die beschriebenen Charakteristika eindeutig zu den genannten Marktstrukturen führen oder ob nicht auch gegenläufige Tendenzen existieren. Den positiven Netzwerkeffekten, d. h. dem höheren Nutzen des einzelnen Teilnehmers bei Ausweitung des Teilnehmerzahl, und der Tendenz zu Monopolen können auch Nachteile einer größeren Netznutzung entgegenstehen, wie z. B. die Überfüllung der Netzwerke, die höheren Kosten ihrer Organisation oder dadurch, dass mit der einheitlichen Netzwerkleistung unterschiedliche Präferenzen nicht befriedigt werden. Verschiedenste technische und ökonomische Gegebenheiten in den vielfältigen Bereichen der „digitalen Ökonomie“ erlauben demnach keine allgemeinen Schlussfolgerungen hinsichtlich Marktstrukturen, abgeleitetem „Marktversagen“ und Regulierungsnotwendigkeiten. An diesen Stellen wird leicht weiterer Forschungsbedarf deutlich, mit dem differenziertere Aussagen getroffen werden könnten.

\section{BÜCHER KURZ VORGESTELLT}

\author{
ORIO GIARINI, WALTER R. STAHEL: Die \\ Performance-Gesellschaft: Chancen und \\ Risiken beim Übergang zur Service Econo- \\ my. Marburg: Metropolis Verlag, 2000. \\ 320 S. DM 39,80. ISBN 3-89518-320-2
}

Dieses Buch handelt von Wirtschaft und Arbeit; es analysiert die Entwicklung der letzten zweihundert Jahre (die Industriegesellschaft) und zeigt mögliche Zukünfte (die Dienstleistungsgesellschaft), welche der Gesellschaft große neue Chancen bieten, aber auch einen Umbau der Wirtschafts- und Arbeitswelt bedingen, der wieder neue Möglichkeiten, aber auch neue Risiken mit sich bringt.

Zuerst in englischer Sprache erschienen, seither in mehrere Sprachen übersetzt, ist dieser Text in Japan in kurzer Zeit zu einem Referenzwerk für ein nachhaltiges Wirtschaften geworden.

Die Autoren haben ihre langjährige Erfahrung in strategischem Denken, welches sie in Industrie, Forschung und Lehre entwickelt und erfolgreich angewandt haben, in diesem Buch zusammengefasst. Die Chancen der Zukunft liegen in einem nachhaltigen Wirtschaften, welches auf drei Pfeilern aufbaut:

- einem systemischen Denken und einer Optimierung auf Systemebene, welche die heutige Produktoptimierung ablösen werden,

- $\quad$ einem Einbezug des Faktors „Zeit“ in die wirtschaftliche Optimierung, welcher wegführt von der traditionellen Betrachtung der Wirtschaft als Fluss zu einer Betrachtung der Wirtschaft als See,

- einem neuen zentralen Wertbezug der Wirtschaft: der Nutzungswert wird zunehmend den Tauschwert ersetzen, und der Verkauf von Leistung über eine Zeitperiode wird den einmaligen Verkauf von Gütern ablösen. 
In jedem dieser drei Pfeiler liegen neben Chancen auch mögliche Risiken, welche für eine Wirtschaft, die garantierte Leistung verkauft statt neuer Güter, typisch sind. Das Buch zeigt, wie die Gesellschaft mit diesen Risiken umgehen kann, ja wie Risiken in Chancen umgewandelt werden können: Das „Problem“ der alternden Bevölkerung ist ein Beispiel dafür.

Die Autoren zeigen, dass diese Chancen nicht nur zu neuen Arbeitsplätzen, sondern auch zu einer höheren Wettbewerbsfähigkeit der Wirtschaft führen. Länder, welche den Umbau zu einem leistungsbezogenen Wirtschaften bewusst fördern, werden in diesem Wettbewerb die Nase vorn haben.

(Verlagsankündigung)

$\gg$

JOCHEN BARTHEL, BERND STEFFENSEN: Koordination im Innovationsprozeß. Standardisierung als Motor des technischen Wandels. Baden-Baden: Nomos Verlagsgesellschaft, 2000. (Forum Kooperative Politik Band 3) 237 S. ISBN 3-7890-6502-1

Der vorliegende dritte Band der Reihe „Forum Kooperative Politik" dokumentiert die überarbeiteten Ergebnisse des Projektes „Förderung freiwilliger Vereinbarungen im Innovationsprozess - entwicklungsbegleitende Normung und Technikfolgenabschätzung“. Dieses Vorhaben wurde an der Akademie für Technikfolgenabschätzung in Baden-Württemberg in enger Kooperation mit dem DIN Deutsches Institut für Normung e.V. in Berlin in der Zeit von April 1996 bis August 1998 durchgeführt.

Innovationen sind für die beteiligten Akteure riskante Vorhaben. Dies gilt vor allem für Zukunftstechnologien, die sich durch technische Komplexität und durch große Anforderungen bei der Positionierung am Markt auszeichnen. Um die bestehenden Risiken zu bewältigen, versuchen Unternehmen ihre Vorhaben abzusichern, indem sie bereits im laufenden Innovationsprozess die Koordination mit potenziellen Nutzern, Zulieferern, Konkurrenten und Behörden suchen.

Im Mittelpunkt des Projekts steht das neue Koordinationsinstrument der entwicklungsbegleitenden Normung (EBN). Es soll dazu bei- tragen, möglichst früh im Innovationsprozess durch eine enge Zusammenarbeit von Industrie, F\&E-Organisationen und Normungsgremien die Rahmenbedingungen für die weitere technische Entwicklung zu vereinheitlichen.

Fallstudien zu den Technikfeldern LaserMaterialbearbeitung, Sicherheit in der Informationstechnik sowie Verfahren zur Sanierung kontaminierter Böden veranschaulichen die Koordinationsanforderungen in Innovationsprozessen, zeigen die Bedingungen auf, unter denen Unternehmen das Instrument der EBN nutzen und verdeutlichen, wie EBN besser auf die Interessen von Technikentwicklern abgestimmt werden kann.

Das diesem Bericht zugrundeliegende Vorhaben wurde mit Mitteln des Bundesministerium für Bildung und Forschung gefördert.

(Verlagsankündigung)

\section{$\ll$}

NICOLE C. KARAFYLLIS: Nachwachsende Rohstoffe. Technikbewertung zwischen den Leitbildern Wachstum und Nachhaltigkeit. Opladen: Leske + Budrich, 2000. (Reihe „Soziologie und Ökologie“"Band 5) 447 S. ISBN 3-8100-2844-4

Die vorliegende Arbeit stellt die gekürzte Fassung der im April 1999 von der Universität Tübingen angenommenen Dissertation im Fach Biologie dar. Sie ist im Zeitraum 1995 - 1998 im interdisziplinären Graduiertenkolleg Ethik in den Wissenschaften entstanden und mit einem Promotionsstipendium der DFG gefördert worden.

Bewertungsmaßstäbe für Umwelttechnologien werden am Beispiel nachwachsender Energieträger kritisch diskutiert. Die leitbildorientierte Technikbewertung wird methodisch verfeinert und um ethische Gesichtspunkte ergänzt. Die Vor- und Nachteile der Technik „Energie aus Biomasse“ werden auf aktueller politischer Grundlage neu bewertet.

Aus dem Inhalt:

- Methodenreflexion: Angewandte Ethik und Technikfolgenabschätzung (TA)

- Nachwachsende Rohstoffe (NR) - Einführung in die technischen und energiewirtschaftlichen Grundlagen 
- Wirtschaftliche und kulturelle Bedeutung von Nachwachsenden Rohstoffen

- Nutzungslinien und Rahmenbedingungen von Nachwachsenden Rohstoffen

- Historischer Hintergrund der Energienutzung aus NR/Biomasse

- Theoretische Grundlagen

- Ethische Argumente im Bereich „Energie aus NR/Biomasse“"

- Diskussionshintergründe und Bewertungsprobleme in den untersuchten Studien

- Die Leitbilder „Wachstum“ und „Nachhaltigkeit"

- Das kontextuelle Schalenmodell der problemorientierten Technikbewertung

- Ausblick: NR als Kulturpflanzen.

(Klappentext)

《 